\title{
A comparative simulation study of different decoding schemes in LDPC coded OFDM systems for NB-PLC channel
}

\author{
Nejwa El Maammar ${ }^{1}$, Seddik Bri ${ }^{2}$, Jaouad Foshi $^{3}$ \\ ${ }^{1,3}$ Electronics Instrumentation and Measurements, Faculty of Sciences and Technologies, \\ Errachidia Moulay Ismail University, Morocco \\ ${ }^{2}$ Material and Instrumentations: MIN, ESTM - Moulay Ismail University, Morocco
}

\begin{tabular}{l}
\hline Article Info \\
\hline Article history: \\
Received Dec 6, 2018 \\
Revised Jan 21, 2019 \\
Accepted Mar 3, 2019 \\
\hline
\end{tabular}

\section{Keywords:}

Codes short-block length Impulse noise

LDPC

OFDM

PLC

\begin{abstract}
In this work, we study the performance of Low density Parity Check (LDPC) coded orthogonal frequency-division multiplexing (COFDM) systems when they are applied on the short data block of a narrowband (NB) power line communications (PLC) channel; In the modelled system simulations have been performed using different code lengths. It is assumed that the channel has a multipath propagation with two different noise scenarios: AWGN background noise with and without the presence of impulsive noise. Performances of Various soft and hard decision LDPC decoder schemes such as belief propagation (BP), weighted bit flipping (WBF), improved weighted bit flipping (IWBF) and implementation-efficient reliability ratio based weighted bit flipping (IRRWBF) decoders were investigated. It has been shown for all simulations performed in PLC channel model showed that remarkable performance improvement can be achieved by using short-length LDPC codes. Especially, the improvements are striking when the BP decoding algorithms are employed on the receiver side.
\end{abstract}

Copyright $\odot 2019$ Institute of Advanced Engineering and Science. All rights reserved.

\section{Corresponding Author:}

Nejwa EL Maammar,

Electronics Instrumentation and Measurements,

Faculty of sciences and technologies,

Errachidia Moulay Ismail University, Morroco.

Email: nejwa.elmaammar@gmail.com

\section{INTRODUCTION}

Recently, Power line communications (PLC) methods have attracted much interest in the field of communication and smart grid systems [1]. They are being extensively developed and represents an exceptionally promising alternative for high-speed Internet access and home network applications by using the existing power lines.The most significant advantage of the PLC systems when compared with the other wire-line communication systems is that it does not any requirement to establish new communication links since they use the existing power lines for communication purposes [2].

Even though different applications of PLC systems are available in the literature, they can generally be classified into two categories as outdoor and indoor applications [3]. The most popular outdoor applications of PLC technologies are smart grids (SG) and advanced meter reading (AMR). The attention paid to smart grid is rapidly increasing due to several reasons such as remote monitoring, measurement, control processes of conventional and renewable grids in a sustainable and reliable way [5-17]. The broadband (BB) indoor applications of the PLC technologies are predicted to offer a communication platform with a high data rate communication medium for homes and offices use [4-6].

Narrowband (NB) PLC, which is also the research topic in this work, is the evolving PLC technology that occupies a narrow frequency band. NB-PLC has been developed with the scope of offering indoor (home automation) and outdoor (smart grid) command and control services. Example of a PLC network topology shown in Figure 1. 


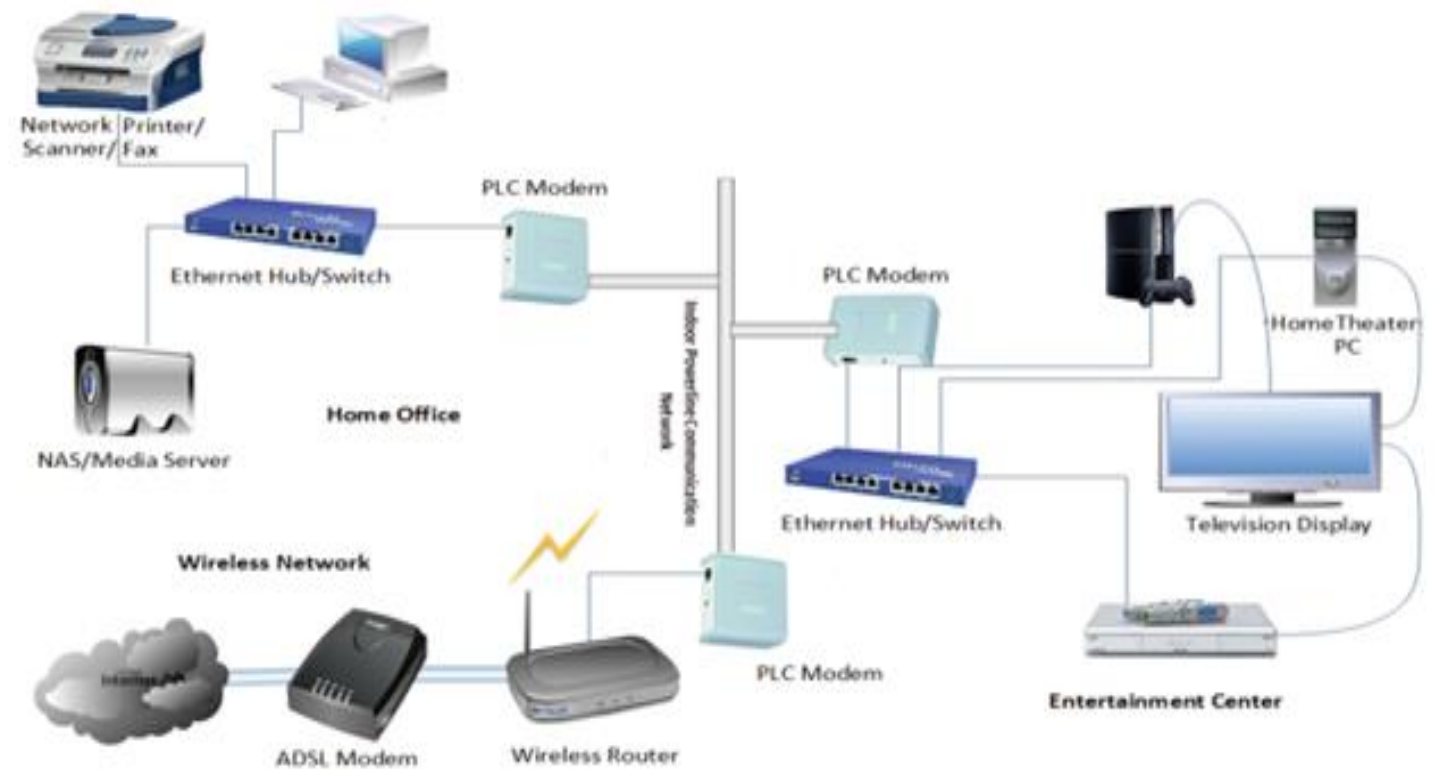

Figure 1. Example of a PLC network topology

The LDPC codes are considered as a candidate scheme for the narrowband PLC in the case when short data blocks are transmitted in many communication channels, such as wireless indoor channels, acoustic channels, and power line channels [7]. In this paper, we study the LDPC codes with different code lengths in the NB-PLC channel. We consider the receiving signal is corrupted by both additive Gaussian noise and impulsive noise. The destructive effects of the PLC channel can be reduced by using LDPC coded OFDM system The effects of soft and hard LDPC decoder schemes such as belief propagation (BP), weighted bit flipping (WBF) and implementation-efficient reliability ratio based weighted bit flipping (IRRWBF) decoders are also examined in the modelled system regarding to the system performance. The rest of this paper is arranged as follows: Section 2 describes the coding and transmission techniques used, while in Section 3 gives the idea about the channel model and noise model used, Section 4 shows the results of simulations, and finally, Conclusions are drawn in Section 5.

\section{CODING AND TRANSMISSION TECHNIQUE}

\subsection{LDPC Codes}

LDPC codes, are known as an important family of error-correction codes has received much attention in wireless communication systems because of its excellent performance in error correction. They were first presented by Gallager [8] in the early 1960s, and were scarcely considered in the three decades that followed due to its computational complexity and limited computational ability of the receiver at that time [9]. After they have taken considerable attention recently due to their Shannon limits performance with belief propagation decoding algorithm [10] they were rediscovered by MacKay and Neal in 1996 [11]. The ldpc codes are currently used for the channel coding on some communication standars. Such as DVB-S2, DVB-T2, DVB-C2, WiMAX (802.16e), Wi-Fi (802.11n) and 10Gbit Ethernet (IEEE 802.3an) due to their high performance. Graphical representation of $(6,3)$ regular LDPC code shown in Figure 2.

LDPC codes require a sparse matrix which is best represented by a bipartite graphs know as Tanner graphs [12]. The parity-check matrix H contains mostly zeros $(0 \mathrm{~s})$ and a few number of one (1s) element. The number of " 1 " bits in a row of the H matrix is called row weight (wr) and, similarly, the number of " 1 ", bits in a column of the $\mathrm{H}$ matrix is called column weight (wc). A typical parity-check $\mathrm{H}$ matrix for a $(6,3)$ regular LDPC code with $w c=2$ and $w r=4$ is given as:

$$
H=\left[\begin{array}{llllll}
1 & 1 & 0 & 1 & 1 & 0 \\
0 & 0 & 1 & 1 & 1 & 1 \\
1 & 1 & 1 & 0 & 0 & 1
\end{array}\right]
$$




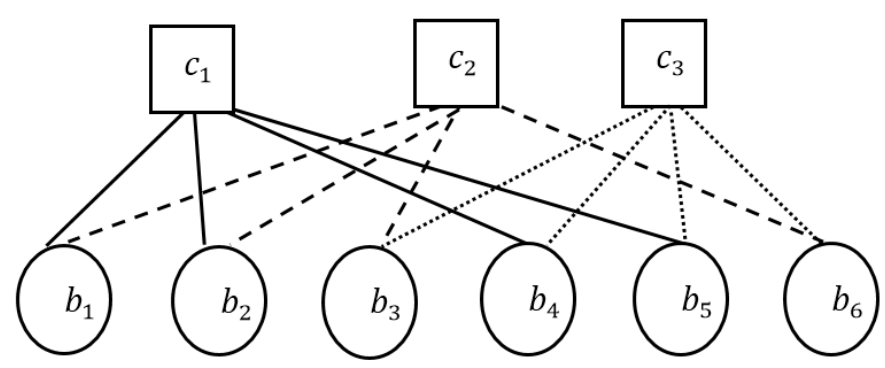

Figure 2. Graphical representation of $(6,3)$ regular LDPC code

Figure 1 illustrate the block diagram of the LDPC coded OFDM system with soft and hard decision decoders employed over PLC channels. At the transmitter side first of all, the randomly generated data is fed into the LDPC encoder block to perform channel-coding task for message bits. The encoded data steam is mapped in the constellation mapper block and then pilot symbols are inserted to the modulated data so as to implement exactly estimation on the receiver side

Afterwards, the data are arranged to add cyclic prefix (CP) in order to prevent intersymbol interference (ISI) and then data are passed through the PLC channels. The data applied to the PLC channels are exposed to different destructive effects of the channel such as attenuation and various noises. The mentioned noise contains background noise, impulsive noise and narrowband interferences.

The remainder blocks following the PLC channel and noise blocks are regarded as the receiver part of the LDPC coded OFDM system. The input data of the receiver are primarly converted to parallel data type and then the guard interval of the parallel data streams are canceled and are applied to the fast Fourier transform (FFT) in OFDM receiver block. By following the FFT process, the FFT computes the fast Fourier transform for the input sequence [13], the data are transormed to serial form. Afterwards, channel estimation and pilot symbols removal processes are performed to serial data stream in the frequency domain. The final step of the receiver system is demodulation and decoding processes. The block diagram of LDPC coded OFDM system with various decoder schemes over PLC channel as sown in Figure 3.

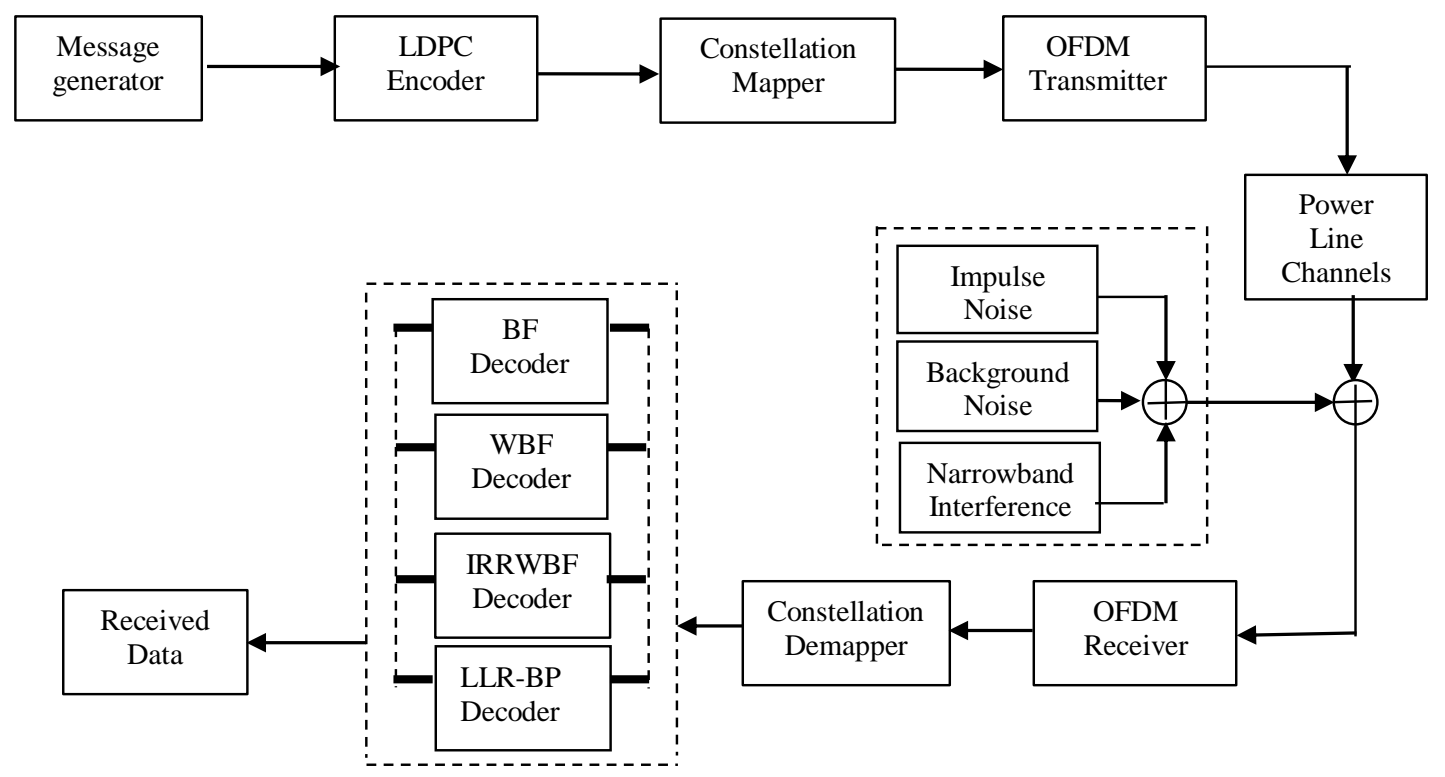

Figure 3. The block diagram of LDPC coded OFDM system with various decoder schemes over PLC channel

\subsection{Decoding Processes}

The LDPC decoding process can be implemented by using either soft or hard decision decoders. The bit flipping (BF) algorithm is usually preferred in hard decision decoders due to its low its low 
complexity. Up to now, several studies have been performed to impove the performance of the BF decoder. Furthermore, modified versions of the BF decoder such as the WBF, IWBF and IRRWBF decoders [14]. The most commonly used soft decoder is belief propagation (BP). In this study, these soft and hard decision LDPC decoder schemes are employed to analyse and to compare the performances over PLC channels.

\subsubsection{Weighted Bit Flipping (WBF) Algorithm}

The WBF algorithm was introduced by Kou et al. [15] to improve the performance of the BF decoding algorithm. The performance of the BF decoding algorithm can be improved by including the the reliability information on the received symbols during the decoding decision steps. as follows:

First of all, in the WBF algorithm, m values are computed by the help of (2) in the WBF algorithm

a) Calculate $\mathrm{S}=\mathrm{H} \cdot \mathrm{v}^{\mathrm{T}}=\left(\mathrm{S}_{0}, \mathrm{~S}_{0}, \ldots \ldots \ldots \mathrm{S}_{\mathrm{M}-1}\right) \quad$ If $S=0$, then terminate the iteration, and declare that the decoding is successful;

b) Find out the most unreliable message node connected to each check node:

$$
|\mathrm{y}| \min -\mathrm{m}=\min _{n: n \in N(m)}\left|y_{n}\right|
$$

Where $N(m)=\left\{n: H_{m n}=1\right\}$ and $m=1,2, \ldots \ldots \ldots, M$. After the determination of $m$ values, the WBF decoding algorithm is performed according to the steps given below:

Step 1: Hard decision sequence $v$ is used for determining syndrome component $S_{m}$.

$$
\mathrm{S}_{\mathrm{m}}=\sum_{\mathrm{n}=1}^{\mathrm{N}} \mathrm{v}_{\mathrm{n}} \mathrm{H}_{\mathrm{mn}}
$$

Step 2: For each message node, compute the error metric $E_{n}$ using $(10)$ for $(n=1,2, \ldots . N)$;

$$
\mathrm{E}_{\mathrm{n}}=\sum_{\mathrm{m} \in \mathrm{M}(\mathrm{n})}\left(2 \mathrm{~S}_{\mathrm{m}}-1\right)|\mathrm{y}| \min -\mathrm{m}
$$

Step 3: Flip the bit $\mathrm{v}_{\mathrm{n}}$ for $n=\arg \max 1 \leq n \leq N E_{n}$. In the event that the algorithm reaches the maximum number of iterations or all the parity check equations are satisfied then the algorithm is ended otherwise the algorithm is repeated from Step 1 to Step 3.

\subsubsection{Improved Weighted Bit Flipping (IWBF) Algorithm}

IWBF algorithm is an improvement one based on the bit nodes information of weighted bit flipping (WBF) algorithm [18]. It measures the reliability of each bit node from the information of check nodes and bit nodes, while the WBF algorithm that only takes advantage of the information from check nodes [19]. For the AWGN channel a simple measure of the reliability of the received vector $y_{n}$ is its magnitude $\left|y_{n}\right|$. The greater the magnitude, the corresponding hard decision $\left|\mathrm{v}_{\mathrm{n}}\right|$ will be.

$$
|\mathrm{y}|_{\min -\mathrm{m}}=\min _{\{\mathrm{n}: \mathrm{n} \in \mathrm{N}(\mathrm{m})\}}\left|y_{n}\right| ; \quad \mathrm{m} \in[1, \mathrm{M}]
$$

IWBF algorithm for each location $\mathrm{n}$ contains the information from both check nodes and bit nodes. The bit to be flipped is the one that has the largest E. In the IWBF algorithm the weighting factor $(\alpha)$ is a real number that is used for the bit message as can be seen from (7). This weighted facor is greater than zero.therefore, when $\alpha=0$ the IWBF algorithm has become the standard WBF. In this case The steps of the IWBF algorithm can be summarized as follows :

Step 1: Syndrome component $S_{m}$ is computed from hard decision sequence v ;

$$
\mathrm{S}_{\mathrm{m}}=\sum_{\mathrm{n}=1}^{\mathrm{N}} \mathrm{v}_{\mathrm{n}} \mathrm{H}_{\mathrm{mn}}
$$

Step 2: $\mathrm{E}_{\mathrm{n}}$ is computed using (7) for $n=1,2, \ldots \ldots \ldots \ldots, N$;

$$
E_{n}=\sum_{m \in M(n)}\left(2 S_{m}-1\right)|y|_{\min -m}-\alpha \cdot\left|y_{n}\right|
$$

Step 3: Flip the bit $v_{n}$ for $n=\arg \max 1 \leq n \leq N E_{n}$ the algorithm repeats from Step 1 to Step 3 until the algorithm reaches the maximum number of iterations or all the parity check equations are satisfied. 


\subsubsection{Implementation-Efficient Reliability Ratio based Weighted Bit Flipping (IRRWBF) Algorithm}

Even though the reliability ratio based weighted bit flipping algorithm is an efficient hard decision decoding algorithm, it requires a long decoding time for the decoding process. The IRRWBF algorithm can be divided into four steps: initialization, check node, variable node and decision steps. The decoding steps of this algorithm can be summarized as follows:

$$
\mathrm{T}_{\mathrm{m}}=\sum_{\mathrm{n}: \mathrm{n} \in \mathrm{N}(\mathrm{m})}\left|\mathrm{y}_{\mathrm{n}}\right|
$$

Step 1: Syndrome component $S_{m}$ is computed from hard decision sequence v ;

$$
\mathrm{S}_{\mathrm{m}}=\sum_{\mathrm{n}=1}^{\mathrm{N}} \mathrm{v}_{\mathrm{n}} \mathrm{H}_{\mathrm{mn}}
$$

Step 2: $\mathrm{E}_{\mathrm{n}}$ is computed using (10) for $(n=1,2, \ldots . . N)$;

$$
\mathrm{E}_{\mathrm{n}}=\frac{1}{\left|\mathrm{y}_{\mathrm{n}}\right|} \sum_{\mathrm{m} \in \mathrm{M}(\mathrm{n})}\left(2 \mathrm{~S}_{\mathrm{m}}-1\right) \mathrm{T}_{\mathrm{m}}
$$

Step 3: Flip the bit $\mathrm{v}_{\mathrm{n}}$ for $n=\arg \max 1 \leq n \leq N E_{n}$. In the event that the algorithm reaches the maximum number of iterations or all the parity check equations are satisfied, the algorithm is ended.

\subsubsection{Belief propagation (BP) Algorithm}

The BP algorithm is one of the most commonly used algorithms in soft decoders; it performs the decoding process as the transfer of information from the bit node to the check node or vice versa. In the transferred information between the nodes there is the possibility of there being 1 or 0 bits of information.

Initialization: The BP decoder computes a priori probability L(ci) in this step by using the following equation:

$$
\mathrm{L}\left(\mathrm{c}_{\mathrm{i}}\right)=\log \frac{P\left(y_{n} \mid x_{n}=0\right)}{P\left(y_{n} \mid x_{n}=1\right)}
$$

Step 1: $\mathrm{L}_{\mathrm{mn}}^{\mathrm{i}}$ is the Log-likelihood ratio (LLR) from the $m$ th check node to the $n$th bit node in the $i$ th iteration. $L_{m n}^{i}$ is updated as follows:

$$
\mathrm{L}_{\mathrm{mn}}^{\mathrm{i}}=\ln \left(\frac{1+\Pi_{n^{\prime} \in N(m) \backslash n} \tanh \left(v_{m n^{\prime}}^{i-1} / 2\right)}{1-\Pi_{n^{\prime} \in N(m) \backslash n} \tanh \left(v_{m n^{\prime}}^{i-1} / 2\right)}\right)
$$

Step 2: $v_{\mathrm{mn}}^{\mathrm{i}}$ and $\mathrm{v}_{\mathrm{n}}^{\mathrm{i}}$ are updated in the second decoding step as follows:

$$
\begin{aligned}
& \mathrm{v}_{\mathrm{mn}}^{\mathrm{i}}=\mathrm{F}_{\mathrm{n}}+\sum_{\mathrm{m}^{\prime} \boldsymbol{\epsilon}(\mathbf{M}(\mathbf{n}) \backslash \mathrm{m}} \mathrm{L}_{\mathrm{m}^{\prime} \mathrm{n}}^{\mathrm{i}} \\
& \mathrm{v}_{\mathrm{n}}^{\mathrm{i}}=\mathrm{F}_{\mathrm{n}}+\sum_{\mathbf{m} \boldsymbol{\epsilon} \mathbf{M}(\mathbf{n})} \mathrm{L}_{\mathrm{mn}}^{\mathrm{i}}
\end{aligned}
$$

Where $v_{m n}^{i}$ is the LLR of the bit $n$ sent from the bit node $n$ to the check node $m$ in the ith iteration, $v_{n}^{i}$ is the a posteriori LLR of the bit computed at each iteration and $F_{n}$ is the LLR of bit $n$ that is calculated from $y_{n}$.

If the algorithm attains the maximum number of iterations or achieves a valid decoding result the algorithm is terminated, otherwise it continues from the beginning.

\section{CHANNEL AND NOISE REPRESENTATION}

\subsection{Channel Model}

PLC channel models differ from the other communication channel models in topology, structure, and physical properties. Numerous reflections are caused at the joints of the network topology due to impedance variations. Factors such as multipath propagation and attenuation are considered when designing a PLC channel model [20]. 
The signal undergoes multipath fading introduced by the channel. The statistical channel model used is similar to that described in [21]. However, the bandwidth used in this case is $0-500 \mathrm{kHz}$, which represents the narrowband PLC channel. The channel frequency response, H(f), of the PLC channel exhibiting L propagation paths can be modelled using Zimmermann and Dostert model as.

$$
H(f)=\sum_{i=1}^{\mathbf{L}} \underbrace{g_{i}}_{\text {Weighting }} \underbrace{e^{-\left(a_{0}+a_{1} f^{k}\right) d_{i}}}_{\text {Attenuation }} \underbrace{e^{-j 2 \pi f^{k}\left(d_{i} / v_{p}\right)}}_{\text {Delay }}
$$

Where gi is the weighting factor uniformly distributed in $[-1,1], \mathrm{a} 0$ and a1 are the attenuation parameters, $\mathrm{k}=1$ is the exponent of the attenuation factor, $\mathrm{a}_{0}=0.3 \cdot 10^{-2}, \mathrm{a}_{1}=4 \cdot 10^{-10} ; \mathrm{d}_{\mathrm{i}}$ is the path length and $\mathrm{d}_{\mathrm{i}} / \mathrm{v}_{\mathrm{p}}=\tau \mathrm{i}$ is the path propagation delay, $\mathrm{v}_{\mathrm{p}}=2 \cdot 10^{8}$ is the phase velocity of the wave.

\subsection{Noise Model}

The noise present in the PLC channel is considered to be comprised of two components: the background and the impulsive noise. The background noise is still considered AWGN. On the other hand, the impulsive noise component is thought to occur in bursts; the sequence of impulse bursts is defined by three parameters where $d_{m}$ is the impulse duration $A_{m}$ is the burst power amplitude and $\Delta t_{m}$ is the interarrival time within impulses respectively. The amplitude is considered to be Gaussian with zero mean.

In the literature, Middleton's Class A noise model is introduced into a statistical model of impulsive noise environment, which is composed of sum of Gaussian noise and impulsive noise. The PDF of the noise amplitude $\mathrm{z}$ is as follows [22]:

$$
P(z)=\sum_{m=0}^{\infty} \frac{e^{-A} A^{m}}{m} \cdot \frac{e^{-\left(z^{2} / 2 \sigma_{m}^{2}\right)}}{\sqrt{2 \pi \sigma_{m}}} \text { With } \sigma_{m}^{2}=\sigma^{2} \cdot \frac{(m / A)+\Gamma}{1+\Gamma}
$$

Where A is the impulsive index; $\Gamma=\sigma_{\mathrm{G}}{ }^{2} / \sigma_{\mathrm{i}}{ }^{2}$ is the GIR (Gaussian-to-impulsive noise power ratio) with Gaussian noise power $\sigma_{\mathrm{G}}{ }^{2}$ and impulsive noise power $\sigma_{\mathrm{i}}{ }^{2}$, and $\sigma^{2}=\sigma_{\mathrm{G}}{ }^{2}+\sigma_{\mathrm{i}}{ }^{2}$.

In this work we assume that the impulse burst amplitude is such that results in a power of impulsive noise $\mathrm{NI}=10 \cdot \mathrm{N} 0$, with $\mathrm{N} 0$ the power of the background noise. It follows that the received signal can be written as:

$$
\mathrm{y}(\mathrm{t})=x(t) * h(t)+n_{i}(t)+n_{B}(t)
$$

Where $n_{i}(t)$ is the impulsive noise and $n_{B}(t)$ is the background which is considered to be AWGN with zero mean and variance N0.

\section{RESULTS AND ANALYSIS}

The simulation has been performed in MATLAB software according to the above described system to evaluate the BER performance of the LDPC encoded OFDM system on message transmission. The transmission is considered on a short data block of a NB-PLC channel and affected by multipath propagation together with two noise scenarios are considered: background AWGN noise with and without the presence of impulsive noise. All simulations are carried out by using regular LDPC codes with $(273,191)$ $(96,48)$ and $(1080,540)$ code lengths.the LLR-BP decoding algorithm is adopted for iterative soft decision decoding, the hard decision decoders are employed in the simulations and the maximum decoding iteration number is set to 25 for all decoder types. The simulation parameters are listed in Table 1 .

Table 1. Simulation Parameters used to Obtain BER Performances

\begin{tabular}{ll}
\hline Parameter & Value \\
\hline Sizes of parity check matrices & $(1080,540),(273,191),(96,48)$ \\
LDPC code rates & $0.5,0.125$ \\
LDPC decoder schemes & LLR-BP, WBF, IRRWBF \\
Maximum iteration number & 25 \\
Modulation type & OFDM \\
Cyclic prefix length & 0.53 \\
Channel model & PLC channel model \\
Noise types & Background and Impulsive noise \\
& Narrowband interferences \\
\hline
\end{tabular}


The BER performance results of the system with $(273,191),(96,48)$ LDPC codes are shown in Figures 4 and 5. In these Figures we compare the BER performance of LDPC coded OFDM system in terms of signal to noise ratio (SNR) over PLC channel with and without the presence of impulse noise respectively. It is clearly seen that the LLR-BP decoder outperforms other hard decision decoders for all simulations. In Figure 4 The BER performance of $(273,191)$ LDPC coded systemover PLC when we use background AWGN shows $0.5 \mathrm{~dB}$ better performances by comparing to $(96,48)$ LDPC coded system with WBF, IRRWBF decoders in the level of $10^{-2}$. And for Figure 5 we can see that significant performance improvement can be obtained in comparison of WBF and IRRWBF decoding rule is employed in the receiver of LDPC coded system using either background AWGN and impulse noise.

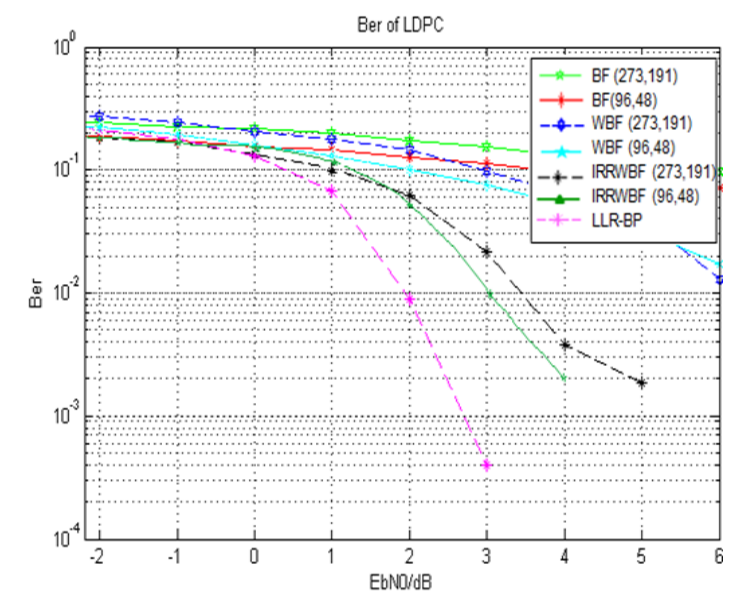

Figure 4. Performance results of the $(273,191)$, $(96,48)$ LDPC codes NB-PLC, noise scenario A (absence of impulsive noise)

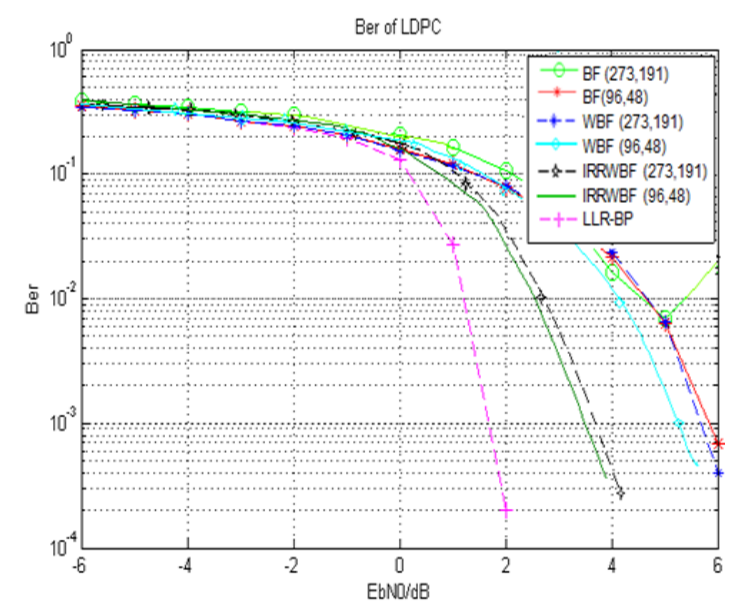

Figure 5. Performance results of the $(273,191)$, $(96,48)$ LDPC codes NB-PLC, noise scenario B (presence of impulsive noise)

In Figures 6 and 7we have compared the BER performance of LDPC coded OFDM system in terms of signal to noise ratio (SNR) over PLC channel with and without presence of impulse noise respectively. In Figure 6 the BER performance of $(1080,540)$ LDPC coded systemover PLC when we use background AWGN shows $0.2 \mathrm{~dB}$ and 1.5 better performances by comparing to $(96,48)$ LDPC coded system with WBF, IRRWBF decoders respectively in the level of $10^{-2}$. And for Figure 7 it has been shown that significant performance improvement can be obtained in comparison of WBF and IRRWBF decoding rule is employed in the receiver of LDPC coded system using either background AWGN and impulse noise.

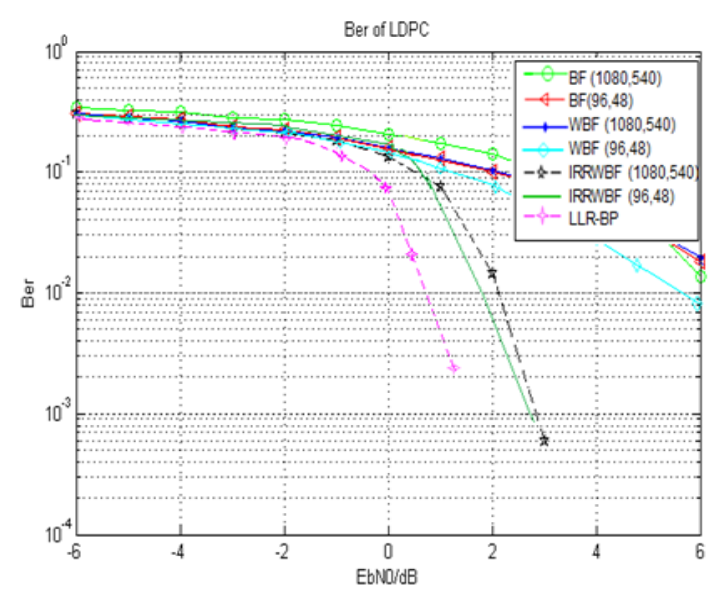

Figure 6. Performance results of the (1080, 540), $(96,48)$ LDPC codes NB-PLC, noise scenario A (absence of impulsive noise)

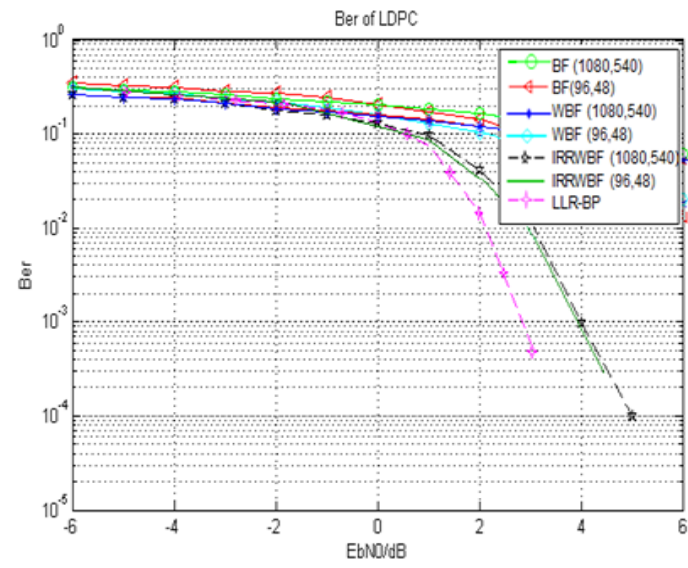

Figure 7. Performance results of the (1080, 540), $(96,48)$ LDPC codes NB-PLC, noise scenario B (presence of impulsive noise) 


\section{CONCLUSION}

In this paper, the BER performance of short LDPC codes for the NB-PLC channel was analyzed for different decoding rules by means of comparative computer simulations. For this purpose different code lengths have been examined. We tested the codes performance in the absence or presence of impulsive noise. The computer simulations were performed for uncoded and LDPC coded OFDM systems that utilize $(1080,540),(96,48)$ and $(273,191)$ LDPC codes. In addition to this, the performances of soft and hard decision LDPC decoders were also investigated regarding to the two different PLC channel scenarios. The performed simulations in the PLC channels showed that the LDPC codes can provide significant improvement with an acceptable encoding complexity when the BP or IRRWBF decoder is utilized on the receiver unit.

\section{REFERENCES}

[1] Y. Kabalci, et al., "LDPC Coded OFDM Systems over Broadband Indoor Power Line Channels: A Performance Analysis," 4th International Conference on Power Engineering, Energy and Electrical Drives, 13-17 May 2013, pp. 1581-1585.

[2] I. Develi, et al., "Performance of LDPC coded image transmission over realistic PLC channels for smart grid applications," Electrical Power and Energy Systems, vol. 62, pp. 549-555, 2014.

[3] I. Develi, et al., "Highly reliable LDPC coded data transfer in home networks by using Canete's PLC channel model," Electrical Power and Energy Systems, vol. 62, pp.912-918, 2014.

[4] A. Majumder, et al., "Power line communications," IEEE Potentials 2004, vol. 4, pp. 4-8.

[5] A.Ipakchi and F. Albuyeh., "Grid of the future," IEEE Power Energy Mag, vol. 7, pp.52-62, 2009.

[6] Y. Lin, et al., "A power line communication network infrastructure for the smart home," IEEE Wireless Commun, vol. 6, pp.104-114, 2002.

[7] N. Andreadou and A. M. Tonello, "Short LDPC Codes for NB-PLC Channel with a Differential Evolution Construction Method," Poceeding of IEEE International Symposium on Power Line Communications and Its Applications, 2013, pp.236-241.

[8] R. G. Gallager, et al., "Low density parity check codes, "IRE Transaction Information Theory, vol. 21, 1962.

[9] N. El Maammar, et al., "Performances Concatenated LDPC Based STBC-OFDM System and MRC Receivers," International Journal of Electrical and Computer Engineering, vol.8, February 2018, pp. 622-630.

[10] W. Ullah, et al., "Comprehensive Algorithmic Review and Analysis of LDPC Codes," TELKOMNIKA Indonesian Journal of Electrical Engineering, vol. 16, October 2015, pp. 111 - 130.

[11] M. Mankar, et al., "Reduced complexity quasi-cyclic LDPC encoder for IEEE 802.11n," International Journal of VLSI design \& Communication System, vol. 7, December 2016, pp. 33-47.

[12] W. Ullah, et al., "QC LDPC Codes for MIMO and Cooperative Networks using Two Way Normalized Min-Sum Decoding, "TELKOMNIKA Indonesian Journal of Electrical Engineering, vol. 12, July. 2014, pp. 5448 -5457.

[13] N. Telagam, et al, "BER analysis of concatenated levels of encoding in GFDM system using labview " Indonesian Journal of Electrical Engineering and Computer Science vol. 14, April 2019, pp. 80-91.

[14] I. Develi, and Y. Kabalci, "A comparative simulation study on the performance of LDPC coded communication systems over Weibull fading channels, " Journal of applied research and technology, vol. 14, pp. 101-107, April 2016.

[15] Julian Webber, et al., "A Study on Adaptive Thresholds for Reduced Complexity Bit-Flip Decoding, "the 14 International Conference on Advanced Communication Technology ICACT, February 2012, pp. 497-501.

[16] M. Korki, et al., "Performance evaluation of a narrowband power line communication for smart grid with noise reduction technique," IEEE Trans Consum Electron, vol. 4, pp.1598-606, 2011.

[17] Y. Kim, et al., "Performance of power line communication systems with noise reduction scheme for smart grid applications," IEEE Trans Consum Electron, vol. 1, pp. 46-52.

[18] Y. Kou, et al., "Low-density parity-check codes based on finite geometries: are discovery and new results," IEEE Trans. Inf. Theory, vol. 7, pp. 2711-2736, 2001.

[19] W. Zhong-xun, et al., "An Improved IWBF Decoding Algorithm Based on LDPC Codes in the Image Transmission," IEEE International Conference on Wireless Communications, Networking and Information Security, August 2010, pp. 98-101.

[20] N. Al-Hinai, et al., "Transmission of Compressed Image over PLCChannel: A Comparative Study," 5th IEEE GCC Conference \& Exhibition, DOI 10.1109/IEEEGCC.2009.5734261, March 2009.

[21] A. M. Tonello, et al., "Wideband Impulse Modulation and Receiver Algorithms for Multiuser Power Line Communications," EURASIP Journal on Advances in Signal Processing, vol. 2007,14 pages, 2007.

[22] Z. Xu, et al., "A decoding algorithm for low-density paritycheck codes in impulse noise environment, "Third International Conference on Communications and Networking in China, DOI 10.1109/CHINACOM.2008.4685174 August. 2008. 